\title{
Isolation of Pseudomonas species from soil sample for production of Pyoverdine and evaluation of its potential as an antimicrobial agent

\author{
Didhiti Agarwal $^{* 1}$, Dhevang Rokkala ${ }^{* 1}$, Shreyas Tawde ${ }^{* 1}$, Prachi Bhatia ${ }^{* 1}$, Sejal Rathod $^{* 1}$
} \\ *l Department of Biotechnology, University of Mumbai, Kishinchand Chellaram College, Mumbai, Maharashtra, India
}

\begin{abstract}
Article Info

Volume 8, Issue 5

Page Number : 355-361

Publication Issue

September-October-2021

Article History

Accepted : 05 Oct 2021

Published : 14 Oct 2021

Pyoverdine, a fluorescent siderophore that have high- affinity for iron is produced by Pseudomonas putida and Pseudomonas aeruginosa that synthesizes it under iron-deficient growth conditions. Pseudomonas species are often encountered in diverse ecological habitats along side being censurable for nosocomial infections spread round the world. Due to these characteristics, there's a growing interest during this microbe for a spread of uses. One such is the production of Pyoverdine, it influences the uptake of iron, along with eliminating the limited iron availability condition.

Pyoverdine is important for Pseudomonas putida and Pseudomonas aeruginosa to cause acute infections. Intense research and study led to the invention of Pyoverdines being a crucial source of chelating Iron. This study is predicated on Pyoverdine extracted from two different species of Pseudomonas which will act as an antimicrobial agent for various species including Escherichia coli and Staphylococcus aureus. The organism, i.e., Pseudomonas putida and Pseudomonas aeruginosa, were isolated from soil sample using medias like: Cetrimide media, King's B media. It had been confirmed using primary biochemical tests, along side species level identification (MALDI - TOF). Isolation was followed by studying the antimicrobial activity of Pyoverdine on different organisms using antibiotics as standard for the same. Results for the tests were obtained, colonies were observed on specific media and zone of inhibition was observed on Muller Hinton plate. Comparative studies were carried out to find which organism used up Pyoverdine or Pyoverdine - Fe complex without using $\mathrm{FeCl} 3$ as sole standard source. Thus, these compounds can synergize with conventional antimicrobials, forming a simpler treatment with serving as a useful gizmo.
\end{abstract}

Keywords: Pseudomonas putida, Pseudomonas aeruginosa, Pyoverdine, Antimicrobial, Siderophore. 


\section{INTRODUCTION}

To date, different sorts of biosensing elements are used effectively for environmental monitoring. Microbial cells seem to be well-suited for this task: they are cheap, adaptable to variable field conditions and provides a measurable response to a broad number of chemicals. Among different pollutants, heavy metals are still a serious problem for the environment. an inexpensive start line for the choice of a bio-recognition element to develop a biosensor for metals might be that of a microorganism that exhibits good mechanisms to deal with metals.[1]

Despite advances in antimicrobial chemotherapy, multi-drug resistant bacteria continue to cause lifethreatening infections, especially in hospitals and with immunocompromised patients.

To drive contamination in these divergent conditions, Pseudomonas uses an assorted armamentarium of harmfulness factors, incorporating those engaged with colonization and disease, supplement procurement, and different modulators of host reaction. Pseudomonads were noticed from the get-go throughout the entire existence of microbial science. Pseudomonas is a variety of Gram- positive, high impact gammaproteobacteria, having a place with the family Pseudomonadaceae authenticating a lot of metabolic variety, and thusly can colonize a wide scope of specialties. [2]

The best studied species include Pseudomonas aeruginosa in its role as an opportunistic human pathogen, the plant Pathogen Pseudomonas syringae, the soil bacterium Pseudomonas putida due to their omnipresent rate in water and plant seeds. Pseudomonas aeruginosa along with Pseudomonas putida are particularly pernicious pathogen [3] as they possess several innate defence mechanisms against antibiotics.

Pseudomonas aeruginosa is one of the main organisms liable for drug-resistant nosocomial infections, and is one among the leading causes of bacteremia and pneumonia in hospitalised patients. While Pseudomonas putida as compared to Pseudomonas aeruginosa has low pathogenic potential but developed immune to an outsized number of antimicrobial agents [4]

Later following quite a while of exploration and extraordinary review novel jobs for a considerable length of time harmfulness determinants are as yet being intuited. Pyoverdine, neither old nor new however later, might be a siderophore created by Pseudomonads. Pseudobactins, are an essential gathering of optional metabolites delivered by microorganisms and plants to work with the take-up of iron, [5] which is normally insoluble in most earthbound conditions. They are bacterial items which tie iron and speed up bacterial iron vehicle involving a dihydroxyquinoline subordinate joined to a kind explicit peptide and, generally, a corrosive or amide. Both the chromophores and accordingly the peptide chains are integrated by nonribosomal peptide synthetases (NRPSs) that empower peptide linkage development between amino acids that can not be consolidated through ribosomal union filling in as antecedent of Pyoverdines and are regularly alluded to as ferribactins [6]. Ferribactins of particular species or possibly strains regularly contrast in their arrangement, prompting an outsized kind of Pyoverdines.

Pyoverdine an iron chelating siderophore on the other side behaves as an opportunistic pathogen in terms of infectious diseases. It acts as an antimicrobial 
agent in various ways, it may also help to form extensive biofilms; along with influencing the ability of certain organisms to germinate and develop biofilms themselves. Pyoverdine was studied to check its ability to displace iron from transferrin as it has high affinity towards iron. In biological and non biological aspects it helps in signalling cascades and transport metals.

Siderophores have received much attention in recent years and are gaining commercial significance due to their potential application in various areas of environmental research, including medicine, agriculture (plant-bacteria synergism and biopesticides) and also since they are safer, and do not cause biomagnification and also provide iron nourishment to the yields subsequently advancing plant development [7] . Thusly, such creatures produce certain valuable parts that can be put to incredible use for the take-up of irons just as improvement of people. These substances are normally created by the creature for their own wellbeing from the hunters or for natural selection.

In this manner, studies have shown that substances with antibacterial, antimicrobial or antifungal movement emitted inside the optional digestion of microorganisms may be applied inside the administration of human, creature, and plant sicknesses [8] and will be further developed and created in the close to forthcoming future.

\section{MATERIALS AND METHODS}

Isolation of Pseudomonas. Pseudomonas putida and Pseudomonas aeruginosa were isolated from garden soil. Serial dilutions of the sample was carried out and were bulk seeded on Sterile King's B agar plate respectively. After incubation under aerobic conditions at $37^{\circ} \mathrm{C}$, appropriate colonies were selected, purified, sub-cultured, stored for further studies and subjected to identification tests. $[1,2,9]$
Identification of the isolates: The isolates of the selected strains were identified based on the cultural, morphological and biochemical tests such as Gram staining, sugar fermentation, catalase test and oxidase test, as outlined in Bergey's Manual of Systematic Bacteriology. MALDI-TOF was also carried out. [10] Figure: 1 and 2

Culture medium and Conditions: Following purification, a $2 \mathrm{ml}$ culture (adjusted to 0.1 O.D) was inoculated in $200 \mathrm{ml}$ of Sterile Succinate broth and King's B broth which consisted trace amounts of iron and other metal contaminants and was put on the rotary shaker for 48 hours with a $\mathrm{pH}$ ranging between 7.0 - 7.3. [11] Uninoculated Sterile Succinate broth and King's B broth were used as controls [12]

Extraction of Pyoverdine: A crude extract was obtained by centrifugation and separation. This served as the sample for further qualitative and quantitative tests.[13,8]

Centrifugation: The broths were centrifuged for 5 minutes at $4500 \mathrm{rpm}$. The supernatant was subsequently collected and preserved. It was saturated with $0.02 \mathrm{~g}$ of $\mathrm{NaCl}$.

Separation and Purification: Supernatant along with $50 \mathrm{ml}$ of phenol and chloroform were separated using a separating funnel with treating the organic phase with $200 \mathrm{ml}$ of diethyl ether 20 mins later, two district phases were obtained and the aqueous phase was re-extracted thrice (using the same protocol). [11, $13,15]$. Sample extracts were stored at $4^{\circ} \mathrm{C}$ and was treated with 5\% acidic EDTA Chloroform solution to obtain an iron- free Pyoverdine. The pure Pyoverdine was observed for fluorescence and absorbance was measured.

Siderophore Qualification Tests: The test was carried out using Arnow's method and Tetrazolium test 
method for catecholates and hydroxymates respectively. [16]

Arnow's method: $1 \mathrm{~mL}$ of cell free culture filtrate was mixed with $1 \mathrm{~mL}$ of $0.5 \mathrm{~mol} \mathrm{~L}^{-1} \mathrm{HCl}, 1 \mathrm{~mL}$ of nitritemolybdate reagent and $1 \mathrm{~mL}$ of $1 \mathrm{~mol} \mathrm{~L}^{-1} \mathrm{NaOH}$ and was incubated for $5 \mathrm{mins}$ at $36^{\circ} \mathrm{C}$.

Tetrazolium test method: $1 \mathrm{~mL}$ of culture filtrate was hydrolysed with $0.5 \mathrm{~mL}$ of $1 \mathrm{~N} \mathrm{NaOH}$, and a pich of tetrazolium salt was added. The solution was then mixed with an instant appearance of a deep red colour.

Antimicrobial activity: This activity was carried out on two different organisms that were: Escherichia coli and Staphylococcus aureus thus testing the ability the Pyoverdine for its antimicrobial effect. [17, 18]

Preparation and Plating: 20mL of Sterile $\mathrm{MH}$ butts each containing $1 \mathrm{ml}$ culture (adjusted to 0.5 O.D) was aliquoted in sterile petri plates [19]. Using Agar well diffusion method $50 \mu \mathrm{L}$ of Pyoverdine, Pyoverdine-Fe complex was added with addition of Antibiotics and $\mathrm{FeCl}_{3}$ as standards respectively following incubation at $36^{\circ} \mathrm{C}$ for 24 hours for zone of inhibition.[20]

\begin{tabular}{|l|l|l|}
\hline \multicolumn{1}{|c|}{$\begin{array}{c}\text { Isolate } \\
\text { Characterisitics }\end{array}$} & P. putida & \multicolumn{1}{c|}{ P. aeruginosa } \\
\hline Size & Medium & Small, pin-point \\
\hline Shape & Round & Round \\
\hline Colour & Bue-Green & Yellow-Green \\
\hline Elevation & Convex & Convex \\
\hline Margin & Entire & Entire \\
\hline Consistency & Butyrous & Butyrous \\
\hline Opacity & Translucent & Translucent \\
\hline Gram - nature & Gram negative & Gram negative \\
\hline Morphology & Short rods & Short rods \\
\hline
\end{tabular}

\begin{tabular}{|l|l|l|}
\hline \multicolumn{1}{|c|}{$\begin{array}{c}\text { Biochemical } \\
\text { Tests }\end{array}$} & \multicolumn{1}{c|}{ Standard } & \multicolumn{1}{c|}{ Observed } \\
\hline $\begin{array}{l}\text { Sugar Utilization } \\
\text { Test }\end{array}$ & & \\
- Glucose & - & - \\
• Lactose & - & - \\
• Mannitol & - & - \\
- Xylose & - & - \\
\hline Sucrose & - & - \\
\hline Indole Test & - & - \\
\hline Methyl Red Test & - & - \\
\hline Voges - Proskauer & - & - \\
Test & & \\
\hline Triple Sugar Iron & - & + \\
\hline Test & & \\
\hline Citrate - Utilization & + & \\
\hline Test & + & \\
\hline Urease Test & - & \\
\hline Oxidase Test & + & \\
\hline Rapid Catalase Test & + & \\
\hline
\end{tabular}

Table 2: Biochemical Tests for Pseudomonas Isolates

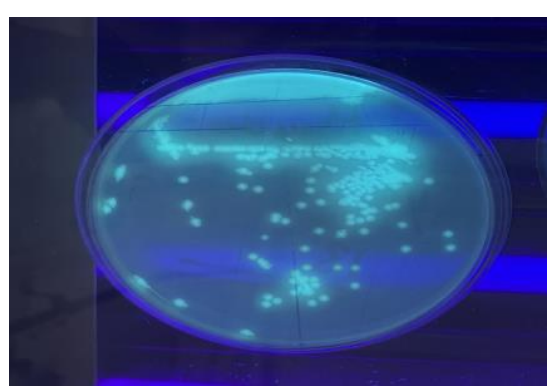

Figure.1: P. putida on Pseudomonas Isolation media

\section{RESULTS AND DISCUSSION}

Identification of Pseudomonas species: Gram staining was carried out along with Catalase test, Sugar Fermentation test, Indole test, Methyl Red 
test, Citrate test, Oxidase test and Voges Proskauer test and, on the basis of the Bergey's Manual of Systemic Bacteriology we can indicate that the isolated culture is Pseudomonas species.

\begin{tabular}{|l|r|r|r|r|}
\hline \multirow{2}{*}{ Wavelength } & \multicolumn{4}{|c|}{ OD of Pyoverdine } \\
\cline { 2 - 5 }$(\mathrm{nm})$ & \multicolumn{2}{|c|}{$P$. putida } & \multicolumn{2}{c|}{$P$. aeruginosa } \\
\cline { 2 - 5 } & $\begin{array}{c}\text { Succinate } \\
\text { Broth }\end{array}$ & $\begin{array}{c}\text { King's B } \\
\text { Broth }\end{array}$ & $\begin{array}{c}\text { Succinate } \\
\text { Broth }\end{array}$ & $\begin{array}{c}\text { King's B } \\
\text { Broth }\end{array}$ \\
\hline $400 \mathrm{~nm}$ & 0.89 & 0.50 & 0.08 & 0.35 \\
\hline $420 \mathrm{~nm}$ & 1.25 & 0.71 & 0.12 & 0.65 \\
\hline $470 \mathrm{~nm}$ & 1.11 & 0.62 & 0.10 & 0.50 \\
\hline $500 \mathrm{~nm}$ & 1.06 & 0.56 & 0.08 & 0.44 \\
\hline $530 \mathrm{~nm}$ & 0.94 & 0.48 & 0.07 & 0.35 \\
\hline $620 \mathrm{~nm}$ & 0.76 & 0.36 & 0.05 & 0.17 \\
\hline $660 \mathrm{~nm}$ & 0.68 & 0.32 & 0.04 & 0.12 \\
\hline $700 \mathrm{~nm}$ & 0.55 & 0.24 & 0.02 & 0.05 \\
\hline
\end{tabular}

Table 3: Absorbance of Fluorescence Quenching Reversal Pyoverdine

\section{Extraction of Pyoverdine:}

\section{$-\infty$ Pseudomonas putida (Succinate broth) \\ $-\rightarrow-$ seudomonas putida (King's B broth) \\ $-\infty$ Pseudomonas aeruginosa (Succinate broth) \\ $-\circ-$ Pseudomonas aeruginosa (King's B broth)}

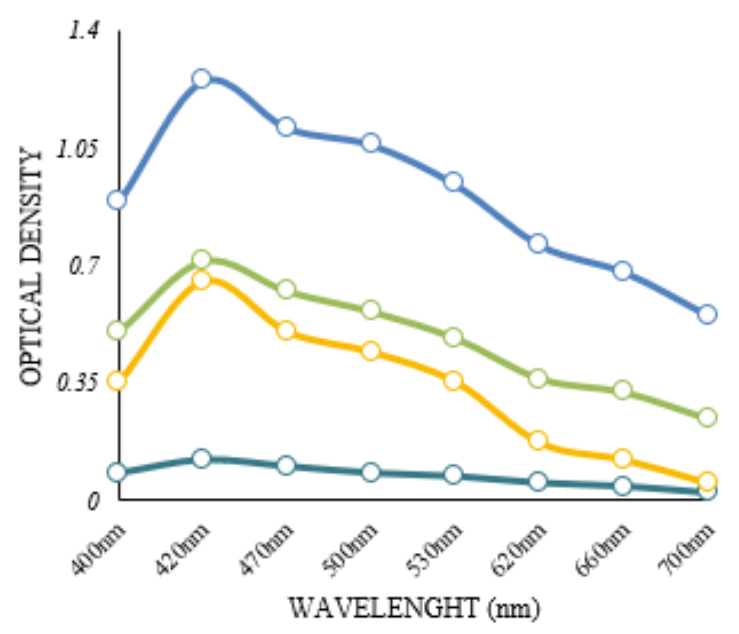

Siderophore Qualification Test: The two methods to detect the type of siderophore present was followed. Arnow's method which detects the presence of catecholate the filtrate resulted to be negative for Pseudomonas species while, Tetrazolium test method detects the presence of hydroxymates resulted to be positive for the species.

\section{Antimicrobial Test:}

When

cell-free

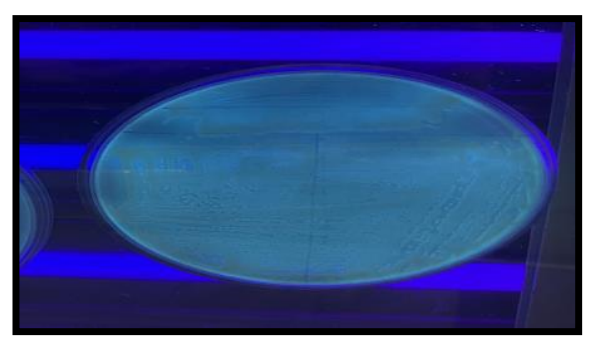

Figure.2: P. aeruginosa on Pseudomonas Isolation media

supernatant that is the Pyoverdine of each culture was applied to the wells of Sterile Muller Hinton agar plates, a zone of inhibition with a diameter of $3.9 \mathrm{~cm}$ was observed around the wells that indicates the uptake of Pyoverdine extracted from succinate broth. Whereas, plates containing Pyoverdine from King's B broth gave no zone of inhibition.

By this It can be confirmed that Pyoverdine utilisation is maximum in the Succinate medium and minimum in King's B broth, since Succinate broth is a synthetic medium it is free of iron and other metal contaminants and thus show greater siderophore activity was not seen contaminants and thus show greater siderophore activity. King's B broth is a non - synthetic rich medium consisting of ingredients like peptone, and beef extract which have iron contaminations and therefore, siderophore activity was not seen and enhanced as compared to Succinate medium.

Graoh 1: Absorbance of Fluorescence Ouenching 


\section{DISCUSSION}

In the past recent years, siderophores and Pseudomonas have grabbed pronounced attention and utmost importance in the field of research around the world. Pseudomonas are one of the microbial type of species unveiling their benefit for glycerol utilisation as well as their physiological, metabolic and genetic features; giving us a larger platform for further research on various other components produced by them for industrial as well as clinical use to the world. The current study undertaken not only focuses on production Pyoverdine but it also portrays a great impact in the field of biotechnology today. Soil is considered to be a sample with maximum number of organisms especially Pseudomonas species which are capable of secreting Pyoverdines essential for iron chelation.

Pseudomonas species isolated from soil was was identified up to the species level and was characterised by Gram staining where Gram-negative, short pink rods were seen. Biochemical tests were in accordance with the Bergey's Manual of Systemic Bacteriology hence, on the basis of these results we can safely say that the isolated organism belongs to Pseudomonas species.

Pyoverdines are yellow-green in colour and are found to be of utmost importance for bacterial virulence and thus they were efficiently extracted from Succinate broth and King's B broth using Diethyl ether, Chloroform and Phenol. Purification was done using 5\% EDTA - Chloroform solution. Separation was based the growth and pigmentation of Pseudomonas putida and Pseudomonas aeruginosa present in the Succinate medium and King's B medium.

Generally, Pseudomonas species studied produce Hydroxymate type of siderophore. Pseudomonas putida and Pseudomonas aeruginosa had a strong reaction to tetrazolium test and no reaction to of the culture. The antimicrobial tests proved that synthetic media are most accurate and the best for siderophore activity. The reason being that media usually have varied levels of iron contamination, carbon and energy source and buffering capacity. Siderophore activity was recorded maximum in Succinate broth and minimum in King's B broth.

Pyoverdine from Succinate broth had maxim buffering capacity on account of di-potassium hydrogen phosphate and potassium di-hydrogen phosphate among the ingredients present. Siderophores are very sensitive to high $\mathrm{pH}$ and so at higher $\mathrm{pH}$ these biomolecules degrade.

It is quite frequent that the level of contaminating iron is already high enough to sustain maximum growth in complex culture media.

\section{CONCLUSION}

The choice of microbial hosts for biotechnological applications had long been based on historical tradition rather than on the bacterial platform which meets the desired measure models in the most ideal manner. This is predominantly in light of the fact that, there are miniature life forms that give out results and are widely researched and portrayed are regularly more handily controlled to expand creation, and become helpful to humankind. One of such creatures incorporate Pseudomonas putida and Pseudomonas aeruginosa that produce siderophores to be specific Pyoverdine.

Pseudomonas species isolated from soil were observed to be equipped for creating Pyoverdine which was extricated and filtered from every one of the stocks and creature. The Pyoverdine detected belonged to the hydroxymate group. The antimicrobial action was checked and both the living beings E.coli and S.aureus utilized Pyoverdine extricated from the Succinate stock giving a zone width of $3.9 \mathrm{~cm}$ while 
King's B broth showed no zone of inhibition. Thus, a manufactured medium will consistently show a more noteworthy action of siderophore on the grounds that it is without iron and sully free while a non engineered medium will contain pollution alongside iron and different metals consequently the movement won't be upgraded and seen.

Thus, with further studies the Pyoverdine can added in antibiotics and be directly inserted into the bacterial host for further and better antimicrobial activity. It will reduce costs along with being a good natural source and be a stronger agent.

\section{ACKNOWLEDGEMENT}

The authors would really like to express sincere gratitude to the DBT Star Status Grant, Jigyaasa Science Honors Program and Kishinchand Chellaram College, Churchgate, Mumbai, Maharashtra, India for giving us this huge platform to work and carry out our research with all the facilities, precautions and safety. The authors also appreciate SRL diagnostics, Mahim, Mumbai, Maharashtra, India for helping us carry out MALDI-TOF.

\section{REFERNCES}

1. Orji Frank Anayo, Ezeanyanso Chika Scholastica, Onyemali Chidi Peter, Ukaegbu Gray Nneji, Ajunwa Obinna and Lawal Oluwabusola Mistura, 2018, The Beneficial Roles of Pseudomonas in Medicine, Industries, and Environment: A Review, DOI: 10.5772/intechopen.85996.

2. Daniel R. Kirienko, Donghoon Kang, and Natalia V. Kirienko, 2018, Novel Pyoverdine Inhibitors Mitigate Pseudomonas aeruginosa Pathogenesis, Front Microbiol. 2018; 9: 3317.

3. Hayder Abdulrahman, Raed Obaid Saleh, 2016, Isolation and Identification of Pseudomonas aeruginosa from different sources (soil, wound, urine) and Checking its MIC with various Antibiotics, Helix Vol.4-5: 795-799 (2016).

4. Dhusi, K. Bajpai, A. Ramteke, P. W., 2018, Overcoming antibiotic resistance: Is siderophore Trojan horse conjugation an answer to evolving resistance in microbial pathogens?, Journal of controlled release: official journal of the Controlled Release Society, 269, 63-87.

5. Iain L. Lamont, Lois W. Martin, 2003, Identification and characterization of novel $\mathrm{P}$ yoverdine synthesis genes in Pseudomonas aeruginosa, Microbiology, volume 149, issue 4 .

6. Mary E. Peek, Abhinav Bhatnagar, 1 Nael A. McCarty, 2 and Susu M. Zughaier, 2012, Pyoverdine, the Major Siderophore in Pseudomonas aeruginosa, Evades NGAL Recognition, Interdisciplinary Perspectives on Infectious Diseases,volume 2012, article ID 843509.

7. Patrick Abou Raji El Feghali and Tarek Nawas, 2018, Extraction and purification of pyocyanin: a simpler and more reliable method, MOJ Toxicol, Volume 4 Issue 6.

8. Joan L. Slonczewski, Kenyon College, 2015, Pseudomonas putida, Microbial Biorealm.

9. Sagar Aryal, 2018, Biochemical Test and Identification of Pseudomonas aeruginosa, Microbiology info. Bergey, D. H., Krieg, N. R., \& Holt,

10. J. G., (1984), Bergey's manual of systematic bacteriology.

11. J.M. Meyer, 1978, The Fluorescent Pigment of Pseudomonas fluorescens Biosynthesis, Purification and Physicochemical Properties, Journal of general Microbiology(1978), issue: 107, pages: 319-328.

12. Angel R. Cueva, Oanh Pham, Aissata Diaby, Derek Fleming, Kendra P. Rumbaugh, and Gregory E. Fernandes, 2020, Pyoverdine Assay for Rapid and Early Detection of Pseudomonas aeruginosa in Burn Wounds, Applied Bio Materials, Volume 3, Issue 8, pages: 4696-5454. 
13. Alok Sharma, Bhavdish N. Johri, 2003, Combat of iron-deprivation through a plant growth promoting fluorescent Pseudomonas strain GRP3A in mung bean (Vigna radiata L. Wilzeck), Microbiological. Research. (2003) Volume: 158, Pages: 77-81 .

14. Patrick Abou Raji El Feghali and Tarek Nawas, 2018, Extraction and purification of pyocyanin: a simpler and more reliable method, MOJ Toxicol, Volume 4 Issue 6.

15. Donghoon Kang, Daniel R. Kirienko, Phillip Webster, Alfred L. Fisher, and Natalia V. Kirienko, 2018, Pyoverdine, a siderophore from Pseudomonas aeruginosa, translocates into C. elegans, removes iron, and activates a distinct host response, Virulence, volume 9, No. 1, pages: 804817.

16. Carlos M. H. Ferreira, Ângela Vilas-Boas, Cátia A. Sousa, Helena M. V. M. Soares and Eduardo V. Soares, 2019, Comparison of five bacterial strains producing siderophores with ability to chelate iron under alkaline conditions, AMB Expr (2019) 9:78 https://doi.org/10.1186/s13568-019-0796-3.

17. Dhusi, K. Bajpai, A. Ramteke, P. W., 2018, Overcoming antibiotic resistance: Is siderophore Trojan horse conjugation an answer to evolving resistance in microbial pathogens?, Journal of controlled release: official journal of the Controlled Release Society, 269, 63-87.

18. Lázaro Molina, Zulema Udaondo, Estrella Duque, Matilde Fernández, Carlos Molina-Santiago, 2014, Antibiotic Resistance Determinants in a Pseudomonas putida Strain Isolated from a Hospital. PLoS One, 2014; 9(1): e81604.

19. Silke Peter, Philipp Oberhettinger, Leonard Schuele, Ariane Dinkelacker, Wichard Vogel, Daniela Dörfel, Daniela Bezdan, Stephan Ossowski, Matthias Marschal, Jan Liese \& Matthias Willmann, 2017, Genomic characterisation of clinical and environmental Pseudomonas putida group strains and determination of their role in the transfer of antimicrobial resistance genes to Pseudomonas aeruginosa, BMC Genomics, volume 18, article no. 859, 2017.

20. Qing-Ping $\mathrm{Hu}$ and Jian-Guo $\mathrm{Xu}, 2011$, A simple double-layered chrome azurol S agar (SDCASA) plate assay to optimize the production of siderophores by a potential biocontrol agent Bacillus, African Journal of Microbiology Research Vol. 5(25), pp. 4321-4327, 9 November, 2011.

\section{Cite this article as :}

Didhiti Agarwal, Dhevang Rokkala, Shreyas Tawde, Prachi Bhatia, Sejal Rathod, "Isolation of Pseudomonas species from soil sample for production of Pyoverdine and evaluation of its potential as an antimicrobial agent", International Journal of Scientific Research in Science and Technology (IJSRST), Online ISSN : 2395-602X, Print ISSN : 23956011, Volume 8 Issue 5, pp. 355-361, SeptemberOctober 2021. Available at doi : https://doi.org/10.32628/IJSRST218555 Journal URL : https://ijsrst.com/IJSRST218555 